\title{
Dispersive versus Dissipative Coupling for Frequency Synchronization in Lasers
}

\author{
Jiajie Ding, ${ }^{1,2}$ Igor Belykh, ${ }^{3}$ Alireza Marandi $\odot,{ }^{4}$ and Mohammad-Ali Miri ${ }^{1,2,{ }^{*}}$ \\ ${ }^{1}$ Department of Physics, Queens College, City University of New York, New York, New York 11367, USA \\ ${ }^{2}$ Physics Program, The Graduate Center, City University of New York, New York, New York 10016, USA \\ ${ }^{3}$ Department of Mathematics and Statistics and Neuroscience Institute, Georgia State University, Atlanta, \\ Georgia 30303, USA \\ ${ }^{4}$ California Institute of Technology, Pasadena, California 91125, USA
}

(Received 24 July 2019; revised manuscript received 8 October 2019; published 15 November 2019)

\begin{abstract}
Coupling-enabled frequency synchronization is essential for an array of light sources operating in a photonic system. Using a two-dimensional nonlinear oscillator model of a laser, we analyze the role of two distinct types of coupling, dispersive and dissipative, in promoting frequency locking between two nonidentical lasers. In both scenarios the two oscillators synchronize into a frequency-locked state when the coupling level exceeds a critical value. We show that the onset of dispersive and dissipative synchronization processes is associated with hard and soft frequency transitions, respectively. Through analysis and numerics, we demonstrate that the dispersive coupling yields bistable synchronization modes, accompanied by asymmetric intensities, and the frequency controlled by the coupling strength. In contrast, dissipative coupling induces monostable synchronization with symmetric intensities and a coupling-independent frequency. Our results are expected to provide a basis for understanding the coupling mechanisms of frequency locking toward controlling synchronization in laser arrays.
\end{abstract}

DOI: 10.1103/PhysRevApplied.12.054039

\section{INTRODUCTION}

Synchronization has been shown to be central for the functioning of a wide spectrum of real-world networks [1-3], ranging from neurons firing together during an epidemic seizure [4] to synchronized power generators [5]. Synchronization is often defined as an adjustment of rhythms in a network of interacting oscillators due to weak interactions. It manifests itself in different ways, including (i) its strongest form of complete synchronization, when all oscillators evolve in unison [6-12], (ii) phase synchronization, which involves phase-locking between the oscillators while their amplitudes may remain unsynchronized [13-16], and (iii) frequency locking or frequency synchronization, when coupled oscillators with different individual frequencies synchronize to a common frequency [17].

The concept of synchronization to a frequency-locked regime is of direct relevance to laser science and engineering. In this context, it is often desired to reach high power levels by coupling a large number of lasers. Clearly, to maintain the coherence properties, such laser arrays should operate in a synchronous regime. This, on the other hand, requires frequency locking of the entire array despite inevitable random detunings of individual elements caused at the fabrication stage as well as

\footnotetext{
*mmiri@qc.cuny.edu
}

postfabrication conditions such as nonuniform pumping, and noise. Therefore, it is of great interest to systematically identify the necessary ingredients for enforcing synchronization in coupled lasers.

The problem of self-induced frequency locking of two coupled lasers has been explored since the early days of lasers. In particular, in early work, Spencer and Lamb [18] explored the dynamics of two Fabry-Perot lasers coupled through a partially transmitting window. In this pioneering work, they showed spontaneous frequency locking of two detuned lasers. In addition, they showed that by controlling the length of a passive cavity, one can tune the oscillation frequency of a coupled laser. Following this work, frequency locking of two coupled lasers was investigated by other authors [19-21]. Despite seemingly simple dynamical equations governing coupled lasers, an analytical expression for the locking threshold was not reported in these studies. In addition, an important aspect that is missing from previous studies is the role of the coupling mechanism in frequency locking. Along different lines, enforcing a stable phase-locked regime in laser arrays is of practical importance since it allows scaling of the irradiance proportional to the square of the number of array elements [22]. In this regard, entrainment of an array of solid-state and semiconductor lasers was explored in previous studies [23,24]. In addition, synchronization of coupled semiconductor lasers operating in a chaotic regime 
was experimentally demonstrated $[25,26]$, and its prospect for secure optical communication was explored [27,28]. Furthermore, self-organized phase locking was experimentally observed in arrays of coupled fiber lasers [29-31]. Most recently, synchronization of two frequency combs was demonstrated in a cascaded arrangement of microring resonators [32].

Given the growing interest in laser-array sources for various applications, including in LIDAR [33], it is critically important to investigate frequency locking in laser arrays. In this regard, it is of interest to understand the role of the underlying processes in synchronization, including the individual lasers involved, the coupling topology in the array, and the strength and type of coupling between each of the two elements. Despite the large body of previous work on synchronization of lasers, less attention has been paid to the last two factors. In this paper, we focus on the role of the coupling mechanism and systematically explore the frequency locking of two lasers, aiming to build a foundation for exploring synchronization through different coupling topologies in larger networks. In this regard, to avoid dealing with the complex chaotic behavior arising in higher dimensionalities of the dynamical variables involved, here we focus on a minimal nonlinear dynamical model for lasers, through which we identify the basic requirements for synchronization.

We consider two different types of interaction between the two lasers: (i) dispersive coupling and (ii) dissipative coupling. According to our results, even though in both cases synchronization occurs for strong coupling levels, these two systems behave fundamentally differently. In both scenarios the dynamics is explored through time-domain simulations, while the stationary states are analytically investigated and their stability is analyzed.

Figures 1(a) and 1(b) schematically depict the interaction of two resonators through dispersive-coupling and dissipative-coupling processes [34]. In the former case, coupling occurs through evanescent tails of the fields in the two laser cavities, while in the latter case it is mediated through an intermediate medium. An exemplary implementation of these two coupling mechanisms is shown in Figs. 1(c) and 1(d) for a pair of microring resonators. When two such resonators are located in close proximity, as shown in Fig. 1(c), light can tunnel from one to the other, with minor leakage to the surroundings. On the other hand, while the evanescent coupling drops significantly for larger separations, a scattering element can be used to mediate the interaction, as shown in Fig. 1(d). Clearly, in this case, the exchange of energy between the two resonators is accompanied by considerable radiation to the surrounding environment.

The implication of these two coupling scenarios is clearly visible in the electric field intensities associated with the eigenmodes of each system shown in Figs. 1(e) and 1(f). As Figs. 1(e) and 1(f) clearly indicate, in the case (a)

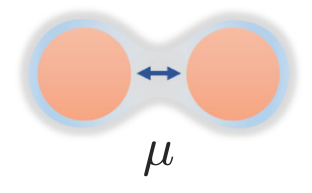

(c)

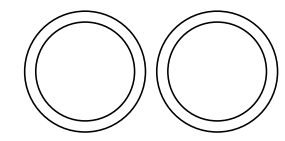

(e)

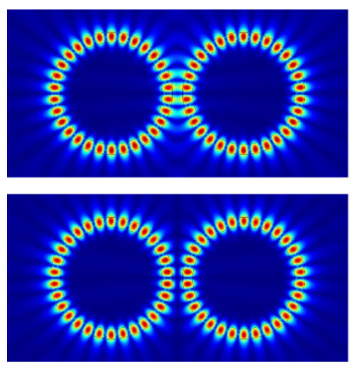

(b)

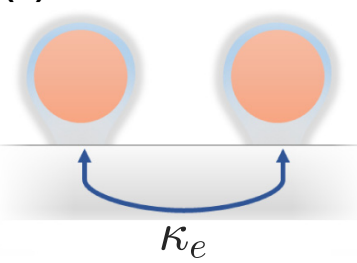

(d)

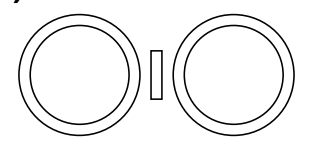

(f)

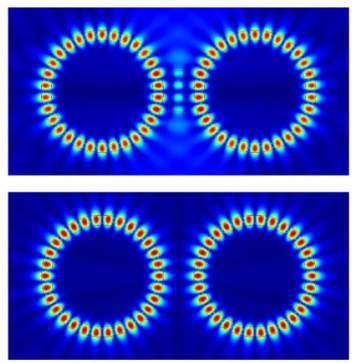

FIG. 1. (a),(b) Two different routes for coupling laser cavities through dispersive coupling (a) and dissipative coupling (b). (c),(d) An exemplary implementation of the two coupling scenarios for a pair of microring resonators. In (c) coupling occurs through the evanescent tails of the fields in the two resonators, while in (d) coupling is mediated through a scattering element, which inevitably causes radiation leakage. (e),(f) The electricfield-intensity profiles of two supermodes associated with the geometries in (c), (d).

of dispersive coupling, the radiation leakages of the two eigenmodes are in similar ranges. In this case both eigenmodes are bound to the microrings despite constructive or destructive interference in the region between the two cavities. In contrast, dissipative coupling creates significant discrimination in the level of the radiative losses of the two eigenmodes. This happens since constructive interference causes a significant overlap of the fields, associated with one of the eigenmodes, with the scattering element, which instead creates large scattering losses. As we discuss in this paper, this contrast between the linear eigenmodes of the two cases of dispersive and dissipative coupling governs the behavior of their nonlinear counterparts, resulting in bistability in one case and monostability in the other. These two scenarios are discussed in Secs. III and IV.

\section{THE NONLINEAR OSCILLATOR MODEL OF A SINGLE LASER}

Depending on the type of the active medium and the laser cavity, various models have been used to describe laser oscillations [35]. A semiclassical treatment of this problem, also known as "neoclassical equations," is 
through solving coupled dynamical equations governing the cavity-mode electric field, the atomic polarization, and the population inversion [35]. However, a great simplification can be made when the atomic variables are adiabatically eliminated. This is a good approximation when the field decay rate is much smaller than the decay rate of the atomic variables (i.e., in a class-A laser) [36]. Under these conditions, laser oscillations can be described through a single equation governing the evolution of the electric field with a proper saturable gain term [37,38]. In this work, we limit our attention to such a model, which we refer to as a "nonlinear oscillator model." It is worth noting that semiconductor lasers are considered to be class-B lasers, where proper modeling requires dynamical interaction between the field and population inversion to be taken into account [39]. However, a class-B laser can also be approximated by a class-A laser when it is operating near the oscillation threshold [36].

In Lamb's self-consistent treatment of a laser oscillator, by considering a cubic dependence of the induced polarization on the electric field, the evolution of the electric field is shown to follow that of the van der Pol oscillator $[37,38,40]$. In this context, the evolution equation governing the slowly varying envelope of the electric field $\mathcal{E}$ is written as $\ddot{\mathcal{E}}+2\left[\kappa-g_{0}\left(1-\varepsilon \mathcal{E}^{2}\right)\right] \dot{\mathcal{E}}+\omega_{0}^{2} \mathcal{E}=0$, where $\omega_{0}$ is the oscillation angular frequency, $\kappa$ represents the linear losses, $g_{0}$ is the linear gain, and $\varepsilon$ is the gain saturation parameter. This simple model is capable of capturing frequency, field, and intensity fluctuation spectra of a laser oscillator [41]. The cubic nonlinear gain introduced in the van der Pol oscillator model, $g=g_{0}\left(1-\varepsilon \mathcal{E}^{2}\right)$, can be considered as a first-order approximation of a saturable gain, $g=g_{0} /\left(1+\varepsilon \mathcal{E}^{2}\right)$ as suggested in Ref. [42]. This saturable gain can also be considered as a first-order Padé approximation of the nonlinear gain term $g=2 g_{0}|\mathcal{E}|^{-2}[1-(1+$ $\left.|\mathcal{E}|^{2}\right)^{-1 / 2}$ ] used by Spencer and Lamb [18].

In addition, given that the resonance frequency is typically much larger than the linear loss and gain, the secondorder equation can be further simplified to a first-order model. As a result, by introducing $a(t)$ as the positive frequency sideband of $\mathcal{E}(t)$, one can suggest the following first-order model for laser oscillations [42]:

$$
\frac{d a}{d t}=\left(-i \omega_{0}-\kappa+\frac{g_{0}}{1+\varepsilon|a|^{2}}\right) a \text {. }
$$

This model was recently used to describe the dynamics of coupled semiconductor microring lasers with uneven pumping in the context of parity-time (PT) symmetry [42] and in optical topological insulators [43]. In this paper, we use this relation to describe laser oscillations, while we interchangeably use the terms "laser" and "oscillator." In the remaining part of this section, we study Eq. (1) before proceeding with coupled lasers.

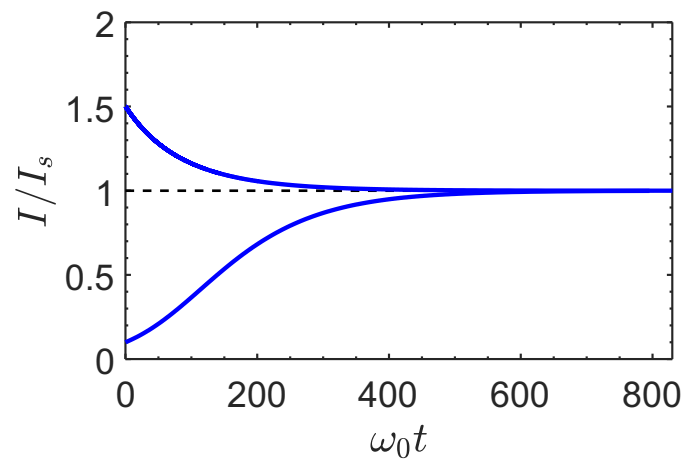

FIG. 2. The analytical solution of the nonlinear oscillator of Eq. (1). The lower and upper branches are associated with initial conditions smaller or larger than the saturation intensity, respectively. In this plot, the parameters are $\kappa=0.01, g_{0}=0.02$, $\varepsilon=1$, and for the lower branch $I_{0}=0.1$ and for the upper branch $I_{0}=1.5$.

The role of the saturable gain in the nonlinear oscillator model described by Eq. (1) can be explained as follows. Initially, for low field intensities, the nonlinearity is weak, and thus $g \approx g_{0}$, which can build up the field, as long as $g_{0}$ is larger than $\kappa$. As the intensity increases, the nonlinear gain decreases until a balance is reached with the linear loss. At this point the field intensity reaches a steadystate value. In the steady state, the field can be described through $a(t)=\sqrt{I_{s}} \exp -i \omega_{0} t$, where the saturation intensity is found by enforcing the condition of gain being equal to loss; that is, $\kappa=g_{0} /\left(1+\varepsilon I_{s}\right)$. Thus, the steady-state field intensity is found to be

$$
I_{s}=\frac{1}{\varepsilon}\left(\frac{g_{0}}{\kappa}-1\right)
$$

On the other hand, the transient behavior of the field intensity, $I=|a|^{2}$, is governed by $\dot{I}=2\left[-\kappa+g_{0} /(1+\varepsilon I)\right] I$, which, under the initial condition of $I\left(t=t_{0}\right)=I_{0}$, admits the following solution:

$$
\frac{I_{0}}{I}\left|\frac{I-I_{s}}{I_{0}-I_{S}}\right|^{g_{0} / \kappa}=e^{-2\left(g_{0}-\kappa\right)\left(t-t_{0}\right)} .
$$

This relation is plotted in Fig. 2 for two different initial conditions, one smaller and the other one larger than the saturation intensity.

\section{COUPLED OSCILLATORS WITH DISPERSIVE COUPLING}

In this section we investigate the dynamics of two coupled oscillators with detuned frequencies. To simplify the analysis, we assume that both oscillators exhibit similar values of gain and loss, and differ only in their frequencies. 
Therefore, the evolution equations can be written as

$$
\begin{aligned}
& \frac{d a_{1}}{d t}=\left(-i \omega_{1}-\kappa+\frac{g_{0}}{1+\varepsilon\left|a_{1}\right|^{2}}\right) a_{1}+i \mu a_{2}, \\
& \frac{d a_{2}}{d t}=\left(-i \omega_{2}-\kappa+\frac{g_{0}}{1+\varepsilon\left|a_{2}\right|^{2}}\right) a_{2}+i \mu a_{1} .
\end{aligned}
$$

It is important to note that in these equations the imaginary coupling describes a dispersive-coupling mechanism. This can be seen by studying the expressions in Eq. (4) in the absence of gain and loss, which results in linear equations $\dot{a}_{1,2}=-i \omega_{1,2} a_{1,2}+i \mu a_{2,1}$ exhibiting real eigenfrequencies

$$
\Omega_{1,2}=\frac{\omega_{1}+\omega_{2}}{2} \pm \sqrt{\left(\frac{\omega_{1}-\omega_{2}}{2}\right)^{2}+\mu^{2}} .
$$

According to this relation, the coupling $\mu$ separates the two eigenfrequencies into real parts, which is why it is referred to as "dispersive coupling." Alternatively, one can call this process "conservative coupling" or "reactive coupling."

Figure 3 depicts the numerical simulations of the dynamics of this system for different coupling levels. According to Fig. 3, in general, the dynamics can be categorized in three different regimes: weak coupling [Figs. 3(a)-3(c)], intermediate coupling [Figs. 3(d)-3(f)], and strong coupling [Figs. 3(g)-3(i)]. In the weak-coupling scenario, the two oscillators behave nearly independently and reach similar steady-state intensities, while they operate at different frequencies. For higher coupling rates, while the oscillation frequencies are modified, multiple harmonics appear at beating frequencies. When the coupling rate is further increased above a critical level $\mu_{c}$, the two oscillators become frequency synchronized and oscillate at a common frequency. In this case, they first evolve interactively and then reach their steady states at different intensities and with a constant phase difference. The frequency-locked regime is associated with a pair of bistable states oscillating at different frequencies. The bistability is expected given that both modes of the coupled-oscillator system have the same oscillation threshold. The excitation of one of the two modes depends on the initial conditions applied.

To better demonstrate the spectral evolution of the system, we evaluate the frequency content of both oscillators versus the coupling level $\mu$ as shown in Fig. 4. In Fig. 4, each line represents a frequency harmonic, while the color gradient represents the strength of that harmonic. According to Fig. 4, the higher-order harmonics, appearing as sidebands, become stronger as the coupling level increases, until the critical coupling value $\mu_{c}$ is reached. When the coupling is further increased above this critical value, all harmonics disappear and only the strongest component survives. This is shown by the thick branches in Fig. 4 .
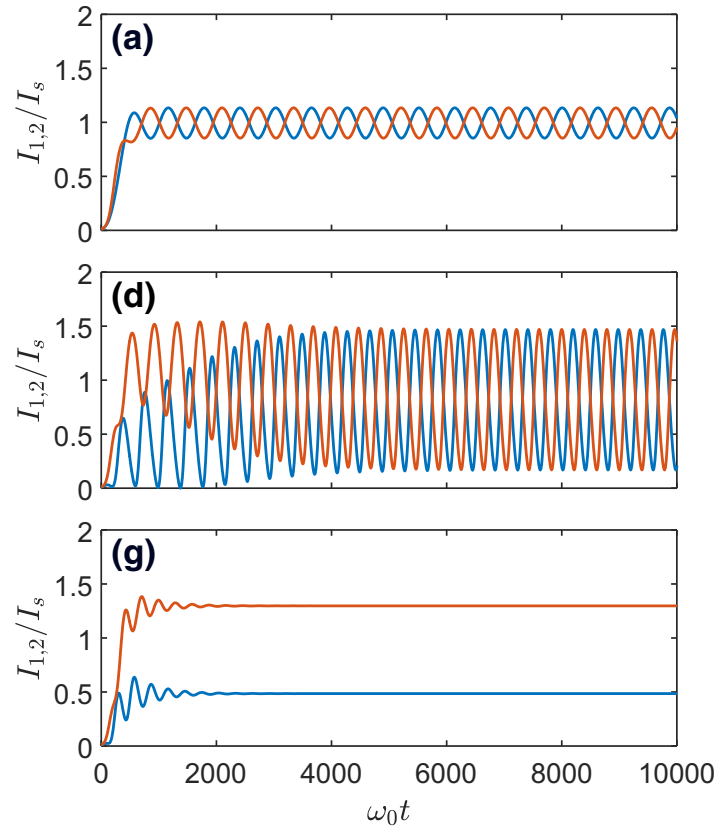
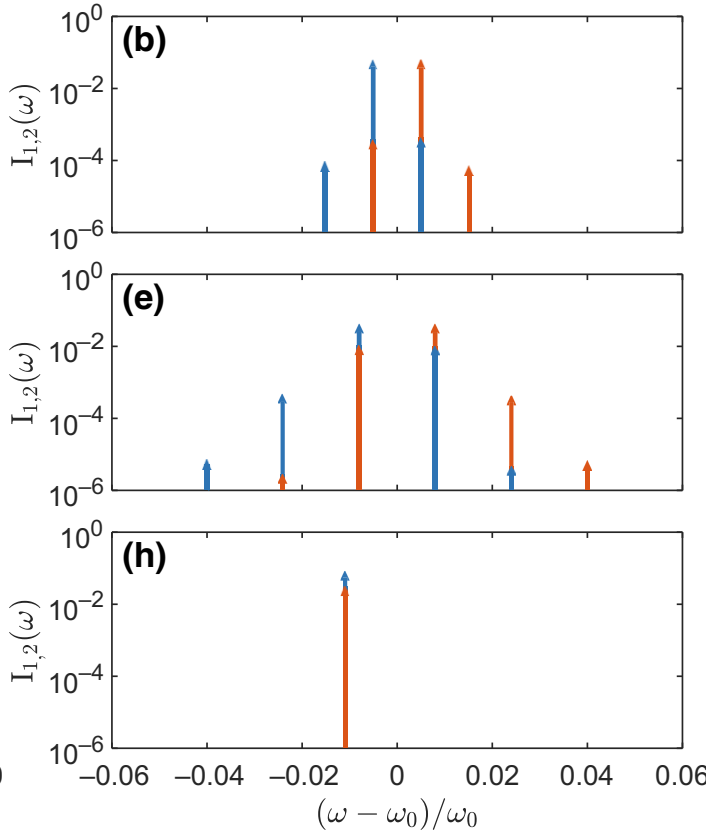
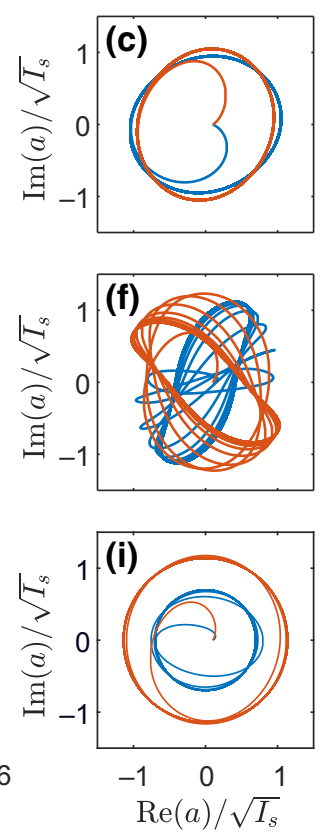

FIG. 3. (a) The time-domain evolution of the intensities of two oscillators with dissimilar frequencies for $\mu=0.001$. (b) The steadystate frequency spectrum of the two oscillators. (c) The phase-space dynamics of the oscillators, where, for clarity, the $a_{1,2}(t)$ terms are divided by a reference phase term, exp $-i \omega_{0} t$, where, $\omega_{0}=\left(\omega_{1}+\omega_{2}\right) / 2$ is the average initial frequency. (d) $-(\mathrm{f})$ The same as parts (a) (c) for $\mu=0.007$. (g)-(i) The same as (a)-(c) for $\mu=0.01$. Here the parameters are $\omega_{1,2}=1 \pm 0.005, \kappa=0.01, g_{0}=0.02$, and $\varepsilon=1$. For these parameters, the critical coupling associated with frequency locking is found to be $\mu_{c} \approx 0.0074$. In all plots, red and blue curves are associated with the first laser and the second laser, respectively. 

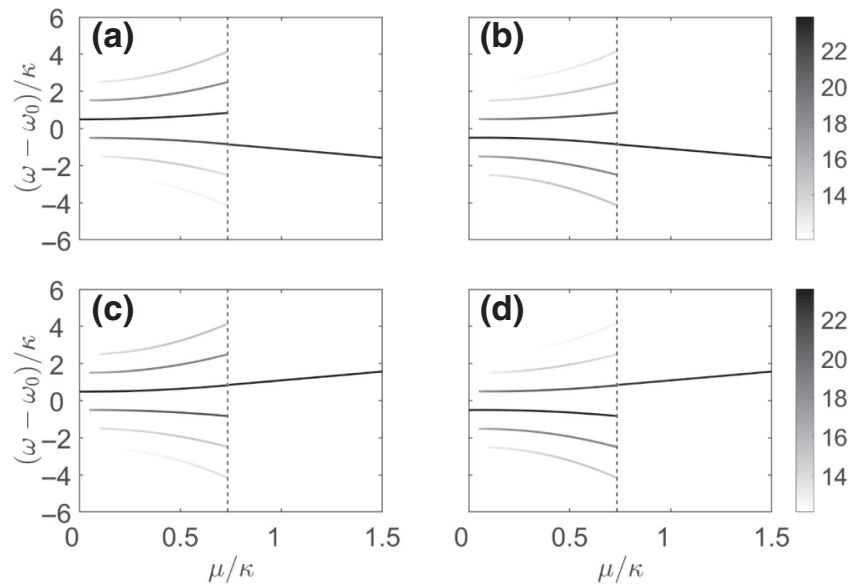

FIG. 4. The frequency spectrum of the two coupled oscillators with dissimilar frequencies versus the coupling strength. In (a),(b) the initial conditions are chosen to initiate operation in the in-phase state, while in (c),(d) they are chosen to initiate operation in the out-of-phase state. The coupling is varied in the range $0 \leq \mu \leq 2 \mu_{c}$, while all other parameters are the same as in Fig. 3. The frequencies are evaluated with respect to the average initial frequency $\omega_{0}=\left(\omega_{1}+\omega_{2}\right) / 2$.

The onset of frequency locking in the initially repelling frequency scenario is an example of the so-called hard (subcritical) transition to the frequency synchronization that is accompanied by the abrupt disappearance of the sideband harmonics. Figures 4(a) and 4(b) and Figs. 4(c) and $4(\mathrm{~d})$ are associated with the two synchronization states appearing as a result of bistability.

It is important to explore the synchronization processes in the parameter space of the system. Considering the form of the expressions in Eq. (4), one can reduce the parameters to three independent variables: $\left(\omega_{1}-\omega_{2}\right) / \kappa, g_{0} / \kappa$, and $\mu / \kappa$. Therefore, the critical coupling level depends on two parameters: $\left(\omega_{1}-\omega_{2}\right) / \kappa$ and $g_{0} / \kappa$. Figure 5(a) depicts the critical coupling versus these two parameters. As expected, by our increasing the frequency detuning, the critical coupling increases. The critical coupling also varies with the ratio of the linear gain to loss; however, this dependency is not as prominent as the effect of detuning. The synchronization region is plotted in the parameter space of detuning-coupling as well as gain-coupling in Figs. 5(b) and 5(c). As expected, the former plot demonstrates a triangular-shaped frequency-locking region, which truly resembles an Arnold tongue [1] given that the vertical axis represents the strength of the influence of each resonator on the other.

Next we focus on the stationary state of the system in the synchronization regime. To investigate the stationary states, we consider an amplitude and phase representation for the complex modal amplitudes; that is, $a_{1,2}(t)=$ $A_{1,2}(t) e^{i \phi_{1,2}(t)}$, where $A_{1,2}(t)$ and $\phi_{1,2}(t)$ are real functions. By defining $\phi(t)=\phi_{2}-\phi_{1}$ as the instantaneous phase

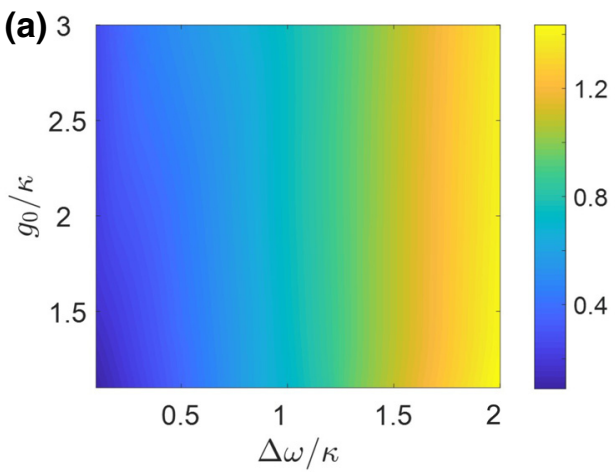

(b)

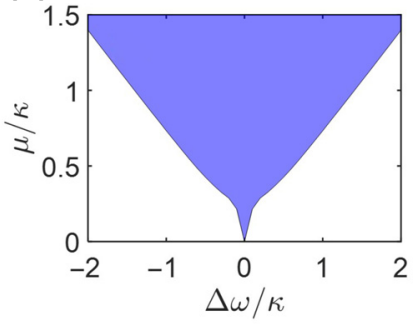

(c)

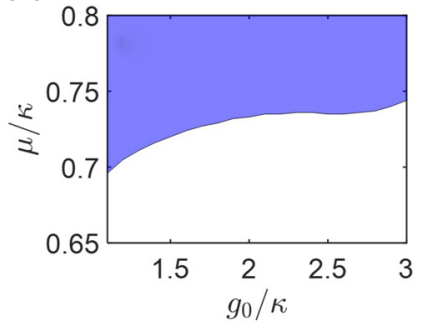

FIG. 5. (a) The critical coupling versus frequency detuning and linear gain. (b) The synchronization region in the parameter map of coupling and frequency detuning for a fixed value of the gain $\left(g_{0} / \kappa=2\right)$. (c) The synchronization region in the parameter map of coupling and linear gain for a fixed value of frequency detuning $(\Delta \omega / \kappa=1)$. All parameters are normalized to the linear loss.

difference between the two oscillators, the expressions in Eq. (4) reduce to three coupled real-valued equations as follows:

$$
\begin{aligned}
\frac{d A_{1}}{d t} & =\left(-\kappa+\frac{g_{0}}{1+\varepsilon A_{1}^{2}}\right) A_{1}-\mu A_{2} \sin \phi, \\
\frac{d A_{2}}{d t} & =\left(-\kappa+\frac{g_{0}}{1+\varepsilon A_{2}^{2}}\right) A_{2}+\mu A_{1} \sin \phi, \\
\frac{d \phi}{d t} & =-\left(\omega_{2}-\omega_{1}\right)+\mu\left(\frac{A_{1}}{A_{2}}-\frac{A_{2}}{A_{1}}\right) \cos \phi .
\end{aligned}
$$

In the frequency-locked regime, the stationary-state solutions can be written as $a_{1,2}(t)=\bar{A}_{1,2} e^{i \bar{\phi}_{1,2}} e^{-i \omega t}$, where $\bar{\phi}_{1,2}$ are constants. Therefore, Eqs. (6a)-(6c) can be written as

$$
\begin{gathered}
\left(-\kappa+\frac{g_{0}}{1+\varepsilon \bar{A}_{1}^{2}}\right) \bar{A}_{1}-\mu \bar{A}_{2} \sin \bar{\phi}=0, \\
\left(-\kappa+\frac{g_{0}}{1+\varepsilon \bar{A}_{2}^{2}}\right) \bar{A}_{2}+\mu \bar{A}_{1} \sin \bar{\phi}=0, \\
-\left(\omega_{2}-\omega_{1}\right)+\mu\left(\frac{\bar{A}_{1}}{\bar{A}_{2}}-\frac{\bar{A}_{2}}{\bar{A}_{1}}\right) \cos \bar{\phi}=0,
\end{gathered}
$$


(a)

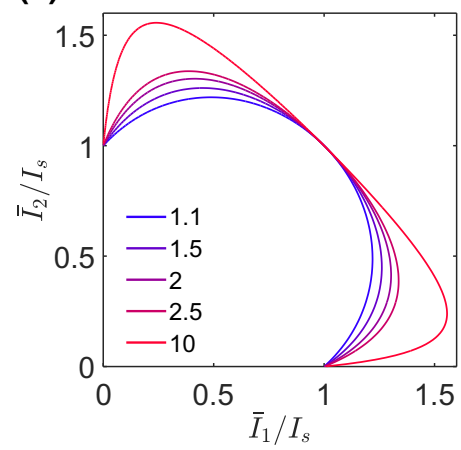

(b)

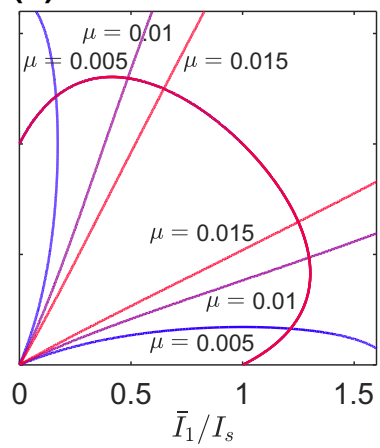

FIG. 6. (a) The contour plots of Eq. (8) showing the stationarystate intensities $\bar{I}_{1,2}$, normalized to $I_{s}$, for different values of the linear-gain-to-loss ratio $g_{0} / \kappa$. (b) Graphical solution of the steady-state intensities as the intersection of graphs corresponding to Eqs. (8) and (9) for three different values of the coupling level.

where $\bar{\phi}=\bar{\phi}_{2}-\bar{\phi}_{1}$ is the phase contrast between the oscillators in the frequency -synchronized regime. By combining Eqs. (7a) and (7b), one can find a relation between the stationary-state intensities, $\bar{I}_{1,2}=\bar{A}_{1,2}^{2}$, as follows:

$$
\left(\frac{g_{0}}{1+\varepsilon \bar{I}_{1}}-\kappa\right) \bar{I}_{1}+\left(\frac{g_{0}}{1+\varepsilon \bar{I}_{2}}-\kappa\right) \bar{I}_{2}=0 .
$$

Figure 6 depicts this relation as a contour in the $\bar{I}_{1}-\bar{I}_{2}$ plane for different values of $g_{0} / \kappa$. As clearly indicated in Fig. 6, one can show that $\bar{I}_{1} \bar{I}_{2}<I_{s}^{2}$, where $I_{s}$ represents the saturation intensity of a single oscillator defined in Eq. (2). Therefore, in the stationary state, while one oscillator operates below $I_{s}$, the other one operates above $I_{s}$. This situation is reversed in the other oscillation mode, recalling that the system is bistable. Equation (8) is independent of the individual frequencies of the two oscillators $\omega_{1,2}$ as well as the coupling strength $\mu$.

Equations (7a)-(7c) can be combined to omit the phase term so as to obtain another identity for the steady-state intensities:

$$
\begin{gathered}
\left(\omega_{2}-\omega_{1}\right)^{2} \frac{\bar{I}_{1} \bar{I}_{2}}{\left(\bar{I}_{1}-\bar{I}_{2}\right)^{2}}-\left(\frac{g_{0}}{1+\varepsilon \bar{I}_{1}}-\kappa\right) \\
\times\left(\frac{g_{0}}{1+\varepsilon \bar{I}_{2}}-\kappa\right)=\mu^{2} .
\end{gathered}
$$

Equation (9) can be considered in conjunction with Eq. (8) to numerically solve for the steady-state intensities. Figure 6(b) depicts a graphical solution of the steadystate intensities obtained as an intersection of these two relations in the $\bar{I}_{1}-\bar{I}_{2}$ plane. It can be shown that in the asymptotic limit of $\mu \gg \mu_{c}$, the stationary-state intensities migrate toward the center point, where $\bar{I}_{1,2} \rightarrow I_{S}$.
In this regime, the nonlinear eigenmodes approach the symmetric and antisymmetric solutions, where the two oscillators operate in phase $(\bar{\phi} \rightarrow 0)$ and in antiphase $(\bar{\phi} \rightarrow \pi)$, respectively. In contrast, when the mutual coupling is slightly above the critical level, the stationary-state intensities are located near the kink and the intensity contrast is large.

The phase difference between the frequency-locked oscillators can be obtained in terms of the intensities from Eq. (7c):

$$
\cos \bar{\phi}=\left(\frac{\sqrt{\bar{I}_{1} \bar{I}_{2}}}{\bar{I}_{1}-\bar{I}_{2}}\right) \frac{\omega_{2}-\omega_{1}}{\mu} .
$$

In this relation the intensities are interchangeable, resulting in two different phase contrasts associated with the two oscillation states. In addition, the synchronization frequency is related to the stationary-state intensities according to

$$
\omega=\frac{\omega_{1}+\omega_{2}}{2}+\left(\frac{\bar{I}_{1}+\bar{I}_{2}}{\bar{I}_{1}-\bar{I}_{2}}\right) \frac{\omega_{1}-\omega_{2}}{2},
$$

which again involves two solutions for the two lasing states. The stationary-state intensities $\bar{I}_{1,2}$, the phase difference $\bar{\phi}$, and the oscillation frequency are depicted versus the coupling strength in Fig. 7. These solutions are meaningful only for $\mu>\mu_{c}$.

\section{COUPLED OSCILLATORS WITH DISSIPATIVE COUPLING}

In this section, we investigate the case of two oscillators coupled through a dissipative mechanism. As mentioned before, this scenario is realized when the energy exchange between two laser cavities is mediated through the surrounding environment, where radiation leakage is inevitable. In this case, the coupled mode equations for the two oscillators are

$$
\begin{aligned}
& \frac{d a_{1}}{d t}=\left(-i \omega_{1}-\kappa+\frac{g_{0}}{1+\varepsilon\left|a_{1}\right|^{2}}\right) a_{1}-\kappa_{e} a_{2}, \\
& \frac{d a_{2}}{d t}=\left(-i \omega_{2}-\kappa+\frac{g_{0}}{1+\varepsilon\left|a_{2}\right|^{2}}\right) a_{2}-\kappa_{e} a_{1} .
\end{aligned}
$$

It is straightforward to show that these equations respect power conservation assuming that both oscillators emit to a common decay channel at rate $\sqrt{2 \kappa_{e}}$. Here the negative sign of the coupling coefficients is due to the in-phase emission of the two oscillators in the decay channel.

It is important to note that $\kappa_{e}$ and $\kappa$ are not independent parameters. Energy conservation demands that dissipative exchange of energy between the two resonators should 

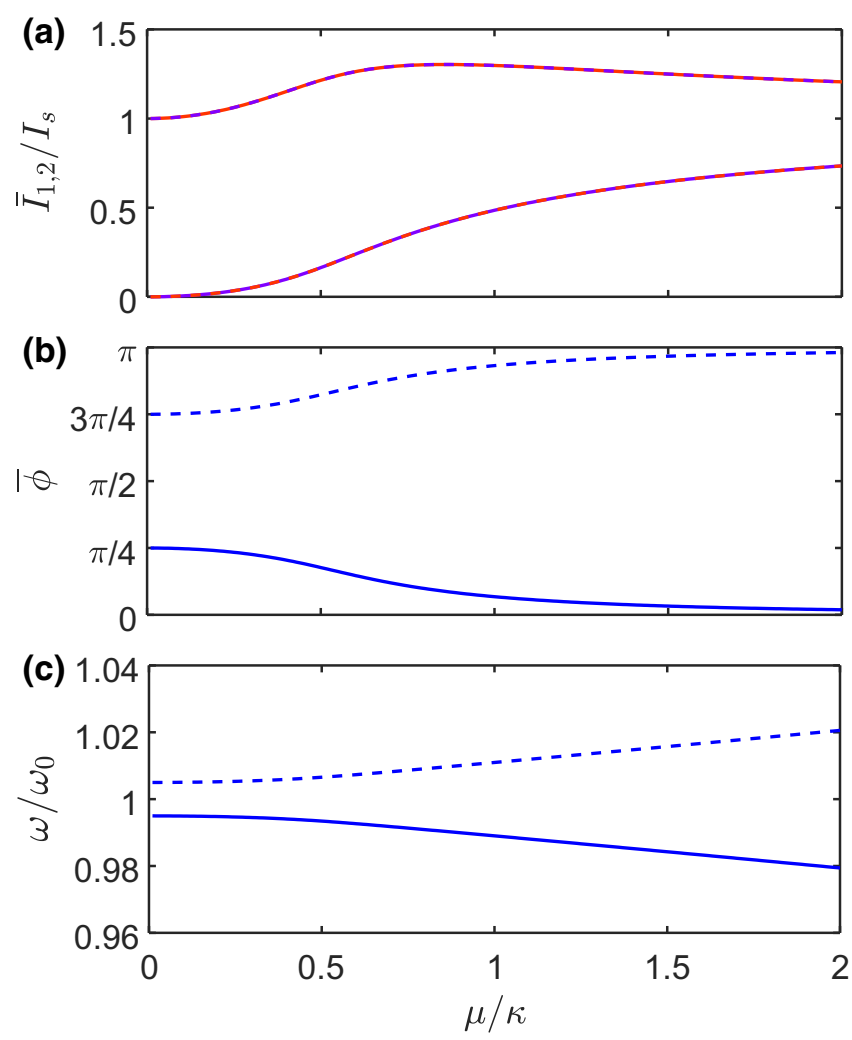

FIG. 7. (a)-(c) The steady state intensities $\bar{I}_{1,2}$, phase difference $\bar{\phi}$, and the oscillation frequency $\omega$ plotted versus the coupling strength $\mu$. In the top panel, blue and red are associated with the intesnity of the two oscillators, while in all panels solid and dashed curves represent the two eigenmodes.

be considered as an additional loss mechanism in each one. Here, for simplicity, we absorb both the intrinsic losses $\kappa_{l}$ and the external losses $\kappa_{e}$ in a single parameter $\kappa=\kappa_{l}+\kappa_{e}$. Therefore, in the expressions in Eq. (12), one should note that $\kappa_{e}<\kappa$. In the absence of intrinsic loss and gain, these equations reduce to a linear system $\dot{a}_{1,2}=\left(-i \omega_{1,2}-\kappa_{e}\right) a_{1,2}-\kappa_{e} a_{2,1}$, which admits two complex eigenfrequencies

$$
\Omega_{1,2}=\frac{\omega_{1}+\omega_{2}}{2}-i \kappa_{e} \pm \sqrt{\left(\frac{\omega_{1}-\omega_{2}}{2}\right)^{2}-\kappa_{e}^{2}},
$$

clearly indicating the dissipative nature of the coupling.

Before investigating the steady-state nonlinear solutions of the expressions in Eq. (12), we explore this system through the time-domain simulations presented in Fig. 8. According to Fig. 8, in this case again the two oscillators synchronize at a common frequency for dissipative couplings above a critical level (i.e., $\kappa_{e}>\kappa_{c}$ ). However, the dynamics is significantly different. The most-prominent effect in this scenario is the tendency for the two oscillators to acquiring the same intensity. This is in stark contrast with the case of dispersive coupling, where symmetric states are asymptotic solutions only for very large coupling rates. Figure 9 depicts the frequency content of the coupled oscillators as a function of the dissipative coupling. At the critical dissipative coupling all frequency harmonics merge, while the synchronization frequency is the average value of the individual frequencies of the two oscillators. As opposed to the previous case, this corresponds to a soft (supercritical) transition to frequency synchronization when the multiple frequency sidebands gradually merge into the synchronization frequency.

In an amplitude and phase representation $a_{1,2}(t)=$ $A_{1,2}(t) e^{i \phi_{1,2}(t)}$, and by our defining the instantaneous phase difference between the two oscillators $\phi(t)=\phi_{2}-\phi_{1}$, the expressions in Eq. (12) can be rewritten as follows:

$$
\begin{aligned}
& \frac{d A_{1}}{d t}=\left(-\kappa+\frac{g_{0}}{1+\varepsilon A_{1}^{2}}\right) A_{1}-\kappa_{e} A_{2} \cos \phi, \\
& \frac{d A_{2}}{d t}=\left(-\kappa+\frac{g_{0}}{1+\varepsilon A_{2}^{2}}\right) A_{2}-\kappa_{e} A_{1} \cos \phi, \\
& \frac{d \phi}{d t}=-\left(\omega_{2}-\omega_{1}\right)+\kappa_{e}\left(\frac{A_{1}}{A_{2}}+\frac{A_{2}}{A_{1}}\right) \sin \phi .
\end{aligned}
$$

In the frequency-locked regime one can write the stationary states as $a_{1,2}(t)=\bar{A}_{1,2} e^{i \bar{\phi}_{1,2} e^{-i \omega t}}$, while, considering $\bar{\phi}=\bar{\phi}_{2}-\bar{\phi}_{1}$ as the steady-state phase difference, one finds the following set of relations:

$$
\begin{aligned}
& \left(-\kappa+\frac{g_{0}}{1+\varepsilon \bar{A}_{1}^{2}}\right) \bar{A}_{1}-\kappa_{e} \bar{A}_{2} \cos \bar{\phi}=0, \\
& \left(-\kappa+\frac{g_{0}}{1+\varepsilon \bar{A}_{2}^{2}}\right) \bar{A}_{2}-\kappa_{e} \bar{A}_{1} \cos \bar{\phi}=0 \\
& -\left(\omega_{2}-\omega_{1}\right)+\kappa_{e}\left(\frac{\bar{A}_{1}}{\bar{A}_{2}}+\frac{\bar{A}_{2}}{\bar{A}_{1}}\right) \sin \bar{\phi}=0
\end{aligned}
$$

By combining Eqs. (15a) and (15b), one finds the following relation between the stationary-state intensities $\bar{I}_{1,2}$ :

$$
\left(\frac{g_{0}}{1+\varepsilon \bar{I}_{1}}-\kappa\right) \bar{I}_{1}=\left(\frac{g_{0}}{1+\varepsilon \bar{I}_{2}}-\kappa\right) \bar{I}_{2}
$$

which can be compared with Eq. (8) in the previous scenario. Clearly, this equation admits a symmetric solution $\bar{I}_{1}=\bar{I}_{2}$. As shown in Fig. 10, however, it also admits an asymmetric branch $\bar{I}_{1} \neq \bar{I}_{2}$. One can show that the asymmetric branch is unstable; therefore, here we focus on the symmetric solution.

Equations (15a)-(15c) simplify greatly when we consider the symmetric branch with $\bar{I}_{1,2}=\bar{I}$. In this case, the 

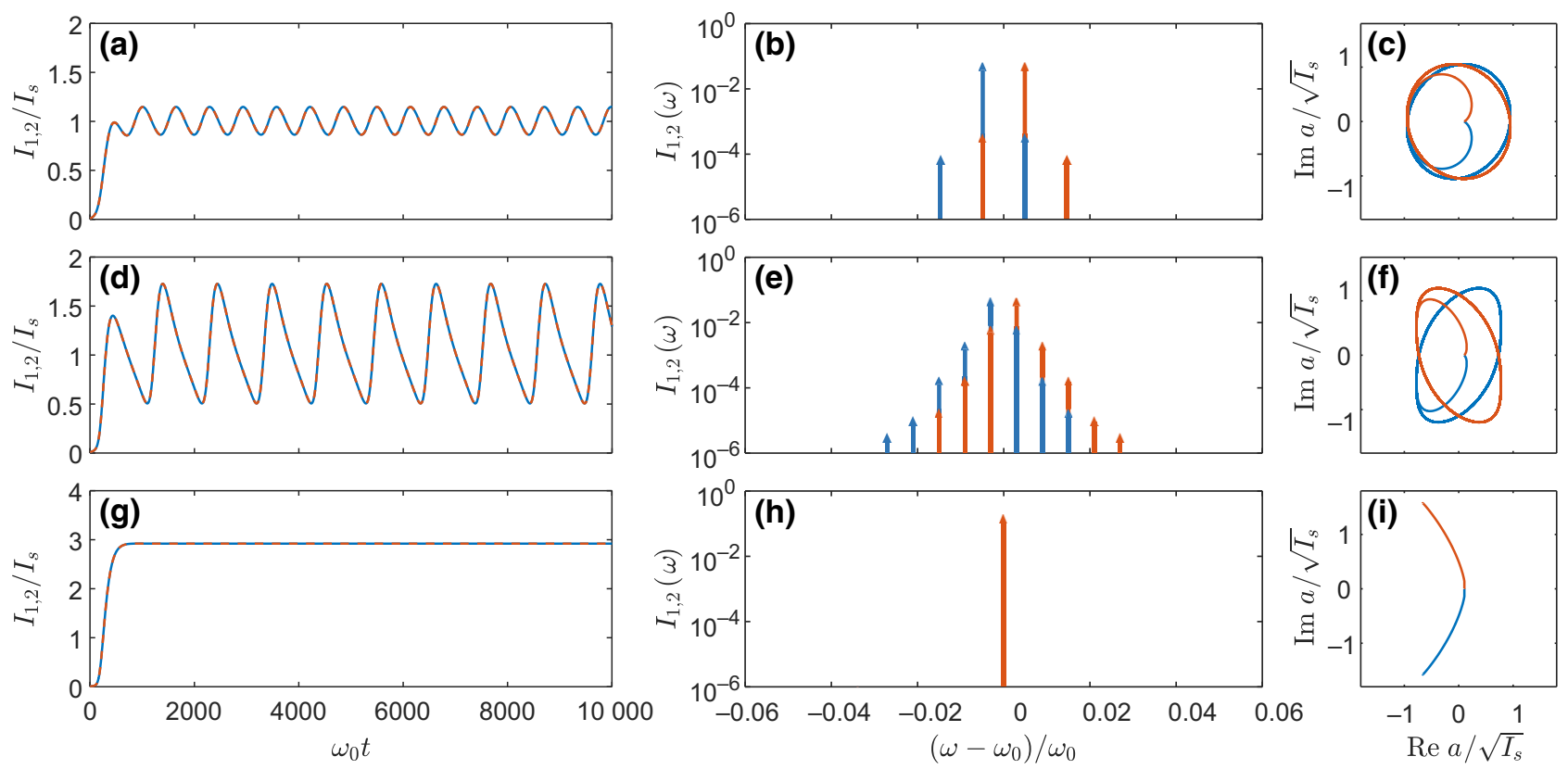

FIG. 8. (a) The time-domain evolution of the intensities of two oscillators with dissimilar frequencies for $\kappa_{e}=0.001$. (b) The steadystate frequency spectrum of the two oscillators. (c) The phase-space dynamics of the oscillators, where, for clarity, $a_{1,2}(t)$ are divided

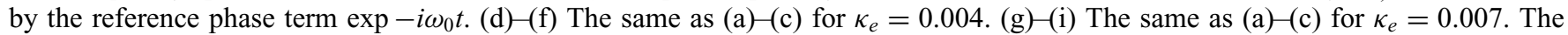
other parameters are $\omega_{1,2}=1 \pm 0.005, \kappa=0.01, g_{0}=0.02$, and $\varepsilon=1$. For these parameters, the critical coupling associated with frequency locking is found to be $\kappa_{c}=0.005$. In all plots, red and blue curves are associated with the first oscillator and the second oscillator, respectively.

stationary-state phase difference between the two oscillators is found through

$$
\sin \bar{\phi}=\frac{\omega_{2}-\omega_{1}}{2 \kappa_{e}}
$$

Therefore, one can simply find a lower bound on the dissipative-coupling rates required for synchronization by considering that the right-hand side should be less than unity. In this manner, the critical dissipative coupling for synchronization is found to be equal to half of the frequency detuning of the two oscillators; that is,

$$
\kappa_{c}=\frac{\left|\omega_{1}-\omega_{2}\right|}{2}
$$

As opposed to the case of dispersive coupling, here the critical coupling is found analytically. The stationary-state intensities of the two oscillators are obtained through Eqs. (15a)-(15c):

$$
\left(\frac{g_{0}}{1+\varepsilon \bar{I}}-\kappa\right)^{2}=\kappa_{e}^{2}-\left(\frac{\omega_{2}-\omega_{1}}{2}\right)^{2}
$$

In addition, the synchronization frequency is found to be the average of the initial oscillation frequencies of the two oscillators:

$$
\omega=\frac{\omega_{1}+\omega_{2}}{2} .
$$

Figures 11(a) and 11(b) depict the stationary-state intensity and phase contrast of the two oscillators in the synchronous regime versus the ratio of the dissipative-coupling rate to the total losses of each oscillator $\kappa_{e} / \kappa$. As governed by Eq. (19), the stationary-state intensity starts from the saturation intensity, $\bar{I}=I_{s}$, at the critical coupling, $\kappa_{e}=\kappa_{c}$, and increases for larger coupling levels. According to (a)

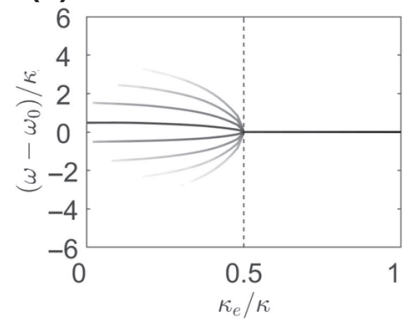

(b)

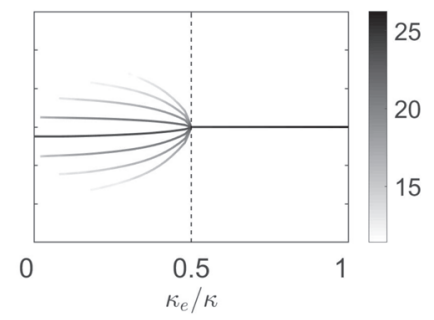

FIG. 9. (a),(b) The frequency spectrum of the two oscillators versus the dissipative coupling rate. Note the soft (supercritical) transition to frequency synchronization when all frequency harmonics gradually merge into the synchronization frequency at the critical coupling rate $\kappa_{e} / \kappa_{c}=0.5$. 


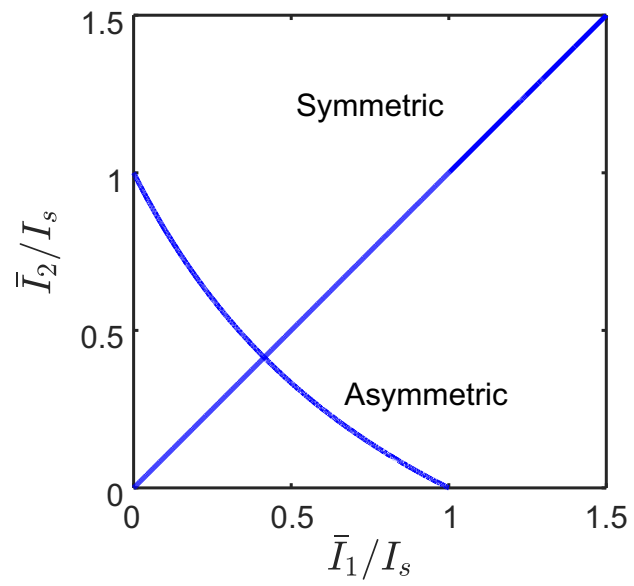

FIG. 10. The symmetric and asymmetric solutions of the steady-state intensities calculated via Eq. (16) for oscillators with dissipative coupling.

Eq. (17), the stationary-state phase difference varies from $|\bar{\phi}|=\pi / 2$ at the synchronization threshold to $\bar{\phi} \rightarrow \pi$ for large dissipative-coupling rates, where the two oscillators become antiphase.

It is worth noting that in case of dissipative coupling the synchronization regime is restricted to a single stable state as opposed to the case of dispersive coupling, where bistability appears. This behavior can be explained through the linear eigenmodes of the coupled oscillators in the absence of gain (i.e., $g_{0}=0$ ). In case of dispersive coupling, the two eigenfrequencies exhibit identical imaginary parts and, thus have equal chances of oscillation when gain is turned on. In contrast, in the scenario of dissipative coupling the two eigenfrequencies exhibit dissimilar losses. In this case, the eigenmode with a smaller imaginary part is protected from radiation to the surrounding environment, and thus can come to oscillation at a lower gain threshold. As a result, bistability is prevented in the case of disspative coupling.

\section{STABILITY ANALYSIS}

Here we perform a linear-stability analysis for the stationary-state solutions obtained in the previous sections. The stability analysis can be done either on the basis of the complex-valued expressions in Eqs. (4) and (12) or through Eqs. (6a)-(6c) and (14a)-(14c), which govern real-valued amplitudes and phases. The latter case offers an advantage given that it deals with fixed-point solutions, thus the stability can be verified simply through the Jacobian-matrix eigenvalues.

First, we consider the case of dispersive coupling. By slightly perturbing the amplitude and phase difference around their fixed-point solutions according to $A_{1,2}=\bar{A}_{1,2}+\alpha_{1,2}$, and $\phi=\bar{\phi}+\varphi$, where $\left|\alpha_{1,2}\right| \ll \bar{A}_{1,2}$ and $|\varphi| \ll|\bar{\phi}|$, one can linearize Eqs. (6a)-(6c) to obtain

$$
\frac{d}{d t}\left(\begin{array}{c}
\alpha_{1} \\
\varphi \\
\alpha_{2}
\end{array}\right)=J\left(\begin{array}{c}
\alpha_{1} \\
\varphi \\
\alpha_{2}
\end{array}\right)
$$

Here $J$ is the Jacobian matrix, with elements defined as $J_{m n}=\partial f_{m} / \partial x_{n}$, where $f_{1}, f_{2}$, and $f_{3}$, respectively, represent the three nonlinear functions on the left-hand side of Eqs. (7a) $-(7 \mathrm{c})$, and $\left(x_{1}, x_{2}, x_{3}\right)=\left(\bar{A}_{1}, \bar{A}_{2}, \bar{\phi}\right)$. The Jacobian matrix takes the form

$$
J=\left(\begin{array}{ccc}
-\kappa+\frac{g_{0}\left(1-\varepsilon \bar{A}_{1}^{2}\right)}{\left(1+\varepsilon \bar{A}_{1}^{2}\right)^{2}} & -\mu \bar{A}_{2} \cos \bar{\phi} & -\mu \sin \bar{\phi} \\
\mu \frac{\bar{A}_{1}^{2}+\bar{A}_{2}^{2}}{\bar{A}_{1}^{2} \bar{A}_{2}} \cos \bar{\phi} & -\mu\left(\frac{\bar{A}_{1}}{\bar{A}_{2}}-\frac{\bar{A}_{2}}{\bar{A}_{1}}\right) \sin \bar{\phi} & -\mu \frac{\bar{A}_{1}^{2}+\bar{A}_{2}^{2}}{\bar{A}_{1} \bar{A}_{2}^{2}} \cos \bar{\phi} \\
\mu \sin \bar{\phi} & \mu \bar{A}_{1} \cos \bar{\phi} & -\kappa+\frac{g_{0}\left(1-\varepsilon \bar{A}_{2}^{2}\right)}{\left(1+\varepsilon \bar{A}_{2}^{2}\right)^{2}}
\end{array}\right) .
$$

Obviously, the fixed-point solutions are stable as long as all the Jacobian-matrix eigenvalues exhibit negative real parts. This criterion is tested by our numerically obtaining the eigenvalues for different values of the coupling rate $\mu$ as shown in Fig. 12(a) for both stationary states. As shown in Fig. 12(a), the largest eigenvalue changes its sign and becomes negative at the critical coupling level. Beyond this value, the stationary-state solutions are stable.

In the case of dissipative coupling, the linearization can be simplified given that the amplitudes of fixed points are symmetric $\bar{A}_{1,2}=\bar{A}$. In this case, the Jacobian matrix is found to be

$$
J=\left(\begin{array}{ccc}
-\kappa+\frac{g_{0}\left(1-\varepsilon \bar{A}^{2}\right)}{\left(1+\varepsilon \bar{A}^{2}\right)^{2}} & \kappa_{e} \bar{A} \sin \bar{\phi} & -\kappa_{e} \cos \bar{\phi} \\
0 & 2 \kappa_{e} \cos \bar{\phi} & 0 \\
-\kappa_{e} \cos \bar{\phi} & \kappa_{e} \bar{A} \sin \bar{\phi} & -\kappa+\frac{g_{0}\left(1-\varepsilon \bar{A}^{2}\right)}{\left(1+\varepsilon \bar{A}^{2}\right)^{2}}
\end{array}\right) .
$$



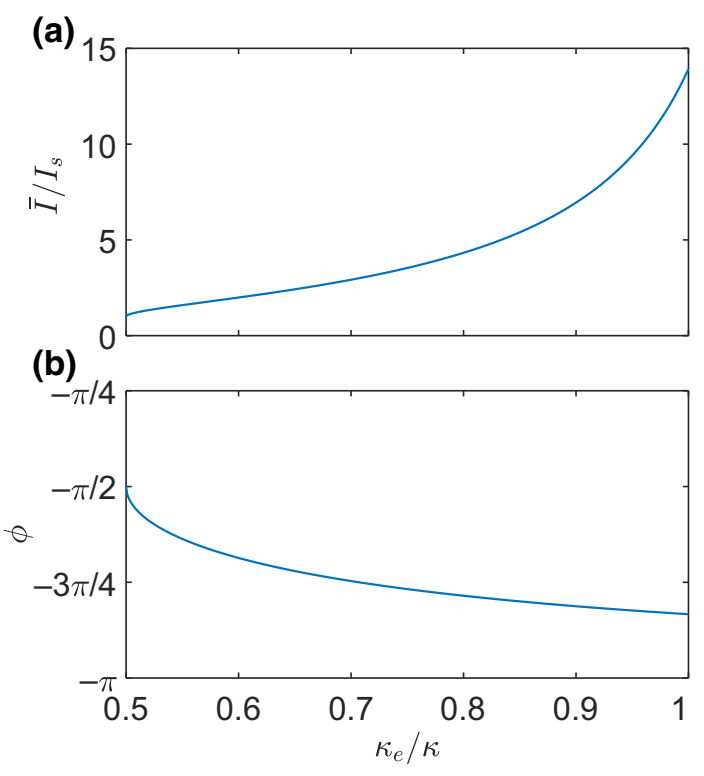

FIG. 11. (a),(b) The stationary-state intensity and the phase contrast between the two oscillators versus the rate of dissipative coupling to total losses of each oscillator. All parameters are the same as in Fig. 8.

It is straightforward to show that the Jacobian matrix admits the following three eigenvalues: $\lambda_{1}, \lambda_{2}=-\kappa \pm$ $\kappa_{e} \cos \bar{\phi}+g_{0}\left(1-\varepsilon \bar{A}_{0}^{2}\right)\left(1+\varepsilon \bar{A}_{0}^{2}\right)^{-2}$ and $\lambda_{3}=2 \kappa_{e} \cos \bar{\phi}$. By use of Eqs. (15a)-(15c), the eigenvalues can be further simplified to $\lambda_{1}=2\left(-\kappa+g_{0}\left(1+\varepsilon \bar{I}_{0}\right)^{-2}\right), \lambda_{2}=$ $-2 g_{0} \varepsilon \bar{I}_{0}\left(1+\varepsilon \bar{I}_{0}\right)^{-2}$, and $\lambda_{3}=-2 \sqrt{\kappa_{e}^{2}-\left(\omega_{2}-\omega_{1}\right)^{2} / 4}$. Clearly, $\lambda_{2}$ is always negative, while, given the condition of synchronization $\kappa_{e}>\kappa_{c}$, and according to Eq. (18), $\lambda_{3}$ is also a negative real number. On the other hand, as shown numerically in Fig. 12(b), the first eigenvalue is also negative for $\kappa_{e}>\kappa_{c}$, indicating that the stationary states are stable.

(a)

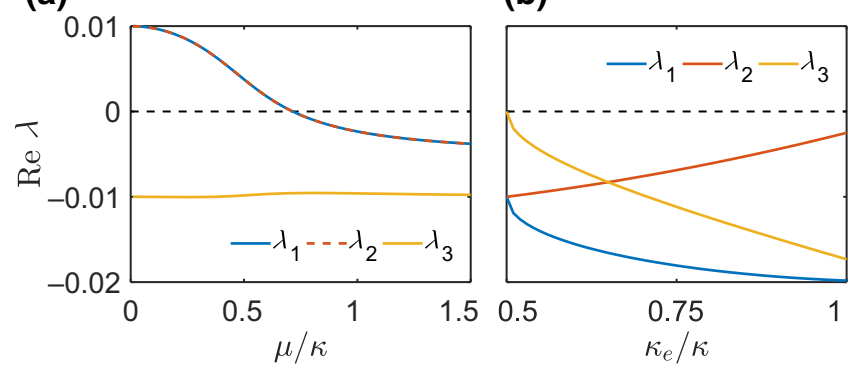

FIG. 12. The Jacobian-matrix eigenvalues associated with the linear-stability analysis of two coupled oscillators with (a) dispersive coupling and (b) dissipative coupling. The parameters are the same as in Figs. 3 and 8. In the case of dispersive coupling, fixed-point solutions exist for all $\mu$ and are stable only for $\mu>\mu_{c}$. In the case of dissipative coupling, fixed-point solutions exist only for $\kappa_{e}>\kappa_{c}$ and are stable in this range.

\section{CONCLUSION}

Through a nonlinear coupled-oscillator model, we investigate the dynamics of two coupled lasers with slightly detuned frequencies. In this discussion, we consider and contrast two separate scenarios of dispersive coupling and dissipative coupling. In both scenarios, when the coupling level exceeds a critical value, the two oscillators undergo a self-organized frequency synchronization. In this regard, we numerically explore the critical coupling and analytically derive general rules for the stationarystate intensities and phases of the two oscillators. The difference between the two scenarios can be summarized as follows. In the case of dispersive coupling, and in the synchronization regime, (i) the system is bistable, (ii) the intensities are asymmetric, and (iii) the frequency depends on the coupling level. In contrast, for the oscillators interacting through dissipative coupling, in the synchronization regime, (i) the system is monostable, (ii) the intensities are symmetric, and (iii) the frequency is the average value of the oscillation frequencies of individual oscillators.

We find that the synchronization properties are deeply rooted in the linear eigenmodes of the coupled-resonator system. In the dispersive-coupling scenario, both eigenfrequencies exhibit similar level of losses, which result in bistable synchronization in the nonlinear regime. In addition, the tendency of the dispersive coupling to repel the eigenfrequencies in the linear regime resists the merging of the two eigenfrequencies through the nonlinear gain, resulting in the hard transition to frequency synchronization. In contrast, in the case of dissipative coupling, the linear eigenmodes are discriminated by their level of losses. Therefore, in the presence of the nonlinear gain, the eigenmode with the smaller linear loss is the only mode to survive. The soft transition to frequency synchronization of the two oscillators in this regime can be understood from the tendency of the dissipative coupling to merge the two eigenfrequencies in the real-frequency domain.

The onset of synchronization in the dissipative-coupling regime is at a point where the two eigenfrequencies collapse, at a so-called exceptional point singularity [44]. This point marks the transition of the two linear eigenfrequencies from an equal to an unequal level of losses. In principle, the coupling between two lasers can be a combination of both the dispersive mechanism and the dissipative mechanism. The results presented here can be easily generalized to this scenario. The relative strength of the two coupling processes rules the behavior of the linear eigenfrequencies, which instead provide insight into the onset of synchronization in the presence of nonlinearities.

Our results provide insight into the synchronization process by identifying the required conditions for frequency locking of two lasers. This work can pave the way for 
future theoretical and experimental investigations on the problem of enforcing global frequency locking in an array of coupled lasers. In this regard, it will be important to use the two different coupling mechanisms investigated here in conjunction with the network topology to design laser arrays that can efficiently self-organize to a common frequency.

\section{ACKNOWLEDGMENTS}

Support for this project was provided by Professional Staff Congress-City University of New York Grant No. 62212-00 50, jointly funded by the Professional Staff Congress and the City University of New York. I.B. was supported by the National Science Foundation under Grant No. DMS-1909924. A.M. acknowledges funding from Army Research Office Grant No. W911NF-18-1-0285 and National Science Foundation Grant No. 1846273.

[1] A. Pikovsky, M. Rosenblum, and J. Kurths, Synchronization: A Universal Concept in Nonlinear Sciences (Cambridge University Press, Cambridge, 2003), Vol. 12.

[2] S. Boccaletti, J. Kurths, G. Osipov, D. Valladares, and C. Zhou, The synchronization of chaotic systems, Phys. Rep. 366, 1 (2002).

[3] A. Arenas, A. Díaz-Guilera, J. Kurths, Y. Moreno, and C. Zhou, Synchronization in complex networks, Phys. Rep. 469, 93 (2008).

[4] T. I. Netoff and S. J. Schiff, Decreased neuronal synchronization during experimental seizures, J. Neurosci. 22, 7297 (2002).

[5] A. E. Motter, S. A. Myers, M. Anghel, and T. Nishikawa, Spontaneous synchrony in power-grid networks, Nat. Phys. 9, 191 (2013).

[6] L. M. Pecora and T. L. Carroll, Master Stability Functions for Synchronized Coupled Systems, Phys. Rev. Lett. 80, 2109 (1998).

[7] J. R. Terry, K. S. Thornburg Jr, D. J. DeShazer, G. D. Van Wiggeren, S. Zhu, P. Ashwin, and R. Roy, Synchronization of chaos in an array of three lasers, Phys. Rev. E 59, 4036 (1999).

[8] V. N. Belykh, I. V. Belykh, and M. Hasler, Connection graph stability method for synchronized coupled chaotic systems, Physica D 195, 159 (2004).

[9] Z. Li and G. Chen, Global synchronization and asymptotic stability of complex dynamical networks, IEEE Trans. Circuits Syst. II 53, 28 (2006).

[10] T. Nishikawa and A. E. Motter, Network synchronization landscape reveals compensatory structures, quantization, and the positive effect of negative interactions, Proc. Natl Acad. Sci. 107, 10342 (2010).

[11] F. Ruiz-Oliveras and A. Pisarchik, Synchronization of semiconductor lasers with coexisting attractors, Phys. Rev. E 79, 016202 (2009).

[12] M. Peil, T. Heil, I. Fischer, and W. Elsäer, Synchronization of Chaotic Semiconductor Laser Systems: Avectorial
Coupling-dependent Scenario, Phys. Rev. Lett. 88, 174101 (2002).

[13] M. G. Rosenblum, A. S. Pikovsky, and J. Kurths, Phase Synchronization of Chaotic Oscillators, Phys. Rev. Lett. 76, 1804 (1996).

[14] D. J. DeShazer, R. Breban, E. Ott, and R. Roy, Detecting Phase Synchronization in a Chaotic Laser Array, Phys. Rev. Lett. 87, 044101 (2001).

[15] S. Boccaletti, E. Allaria, R. Meucci, and F. Arecchi, Experimental Characterization of the Transition to Phase Synchronization of Chaotic $\mathrm{CO}_{2}$ Laser Systems, Phys. Rev. Lett. 89, 194101 (2002).

[16] D. I. Kim, D.-S. Lee, Y.-J. Park, G. U. Kim, and C.-M. Kim, Phase synchronization of chaotic lasers, Opt. Express 14, 702 (2006).

[17] J. A. Acebrón, L. L. Bonilla, C. J. P. Vicente, F. Ritort, and R. Spigler, The Kuramoto model: A simple paradigm for synchronization phenomena, Rev. Mod. Phys. 77, 137 (2005).

[18] M. B. Spencer and W. E. Lamb Jr, Theory of two coupled lasers, Phys. Rev. A 5, 893 (1972).

[19] W. Fader, Theory of two coupled lasers, IEEE J. Quantum Electron. 21, 1838 (1985).

[20] W. W. Chow, Frequency locking in weakly coupled lasers, Opt. Lett. 10, 442 (1985).

[21] H. Mirels, Performance of two coupled lasers, Appl. Opt. 25, 2130 (1986).

[22] A. F. Glova, Phase locking of optically coupled lasers, Quantum Electron. 33, 283 (2003).

[23] Y. Braiman, T. Kennedy, K. Wiesenfeld, and A. Khibnik, Entrainment of solid-state laser arrays, Phys. Rev. A 52, 1500 (1995).

[24] H. Winful and S. Wang, Stability of phase locking in coupled semiconductor laser arrays, Appl. Phys. Lett. 53, 1894 (1988).

[25] T. Sugawara, M. Tachikawa, T. Tsukamoto, and T. Shimizu, Observation of Synchronization in Laser Chaos, Phys. Rev. Lett. 72, 3502 (1994).

[26] R. Roy and K. S. Thornburg Jr, Experimental Synchronization of Chaotic Lasers, Phys. Rev. Lett. 72, 2009 (1994).

[27] V. Annovazzi-Lodi, S. Donati, and A. Scire, Synchronization of chaotic injected-laser systems and its application to optical cryptography, IEEE J. Quantum Electron. 32, 953 (1996).

[28] J.-P. Goedgebuer, L. Larger, and H. Porte, Optical Cryptosystem based on Synchronization of Hyperchaos Generated by a Delayed Feedback Tunable Laser Diode, Phys. Rev. Lett. 80, 2249 (1998).

[29] H. Bruesselbach, D. C. Jones, M. S. Mangir, M. Minden, and J. L. Rogers, Self-organized coherence in fiber laser arrays, Opt. Lett. 30, 1339 (2005).

[30] T. Shay, V. Benham, J. Baker, B. Ward, A. D. Sanchez, M. A. Culpepper, D. Pilkington, J. Spring, D. J. Nelson, and C. A. Lu, First experimental demonstration of selfsynchronous phase locking of an opticalarray, Opt. Express 14, 12015 (2006).

[31] M. Fridman, M. Nixon, N. Davidson, and A. A. Friesem, Passive phase locking of 25 fiber lasers, Opt. Lett. 35, 1434 (2010). 
[32] J. K. Jang, A. Klenner, X. Ji, Y. Okawachi, M. Lipson, and A. L. Gaeta, Synchronization of coupled optical microresonators, Nat. Photonics 12, 688 (2018).

[33] B. Schwarz, Lidar: Mapping the worldin 3d, Nat. Photonics 4, 429 (2010).

[34] J. Ding and M.-A. Miri, Mode discrimination in dissipatively coupled laser arrays, Opt. Lett. 44, 5021 (2019).

[35] A. Siegman, Lasers (University Science Books, Mill Valley, CA, 1986).

[36] J. R. Tredicce, F. T. Arecchi, G. L. Lippi, and G. P. Puccioni, Instabilities in lasers with an injected signal, JOSA B 2, 173 (1985).

[37] W. E. Lamb Jr, Theory of an optical maser, Phys. Rev. 134, A1429 (1964).

[38] A. Yariv, Quantum Electronics (Wiley, 1989).

[39] G. Agrawal and N. Dutta, Long-Wavelength Semiconductor Lasers (van Nostrana Reinhold, New York, 1986).
[40] B. Van der Pol, The nonlinear theory of electric oscillations, Proc. Inst. Radio Eng. 22, 1051 (1934).

[41] A. Yariv and W. Caton, Frequency, intensity, and field fluctuations in laser oscillators, IEEE J. Quantum Electron. 10, 509 (1974).

[42] A. U. Hassan, H. Hodaei, M.-A. Miri, M. Khajavikhan, and D. N. Christodoulides, Nonlinear reversal of the ptsymmetric phase transition in a system of coupled semiconductor microring resonators, Phys. Rev. A 92, 063807 (2015).

[43] G. Harari, M. A. Bandres, Y. Lumer, M. C. Rechtsman, Y. Chong, M. Khajavikhan, D. N. Christodoulides, and M. Segev, Topological insulator laser: Theory, Science 359, eaar4003 (2018).

[44] M.-A. Miri and A. Alù, Exceptional points in optics and photonics, Science 363, eaar7709 (2019). 